\title{
Review of Bone Modifying Agents in Metastatic Breast Cancer
}

Charumathi Raghu Subramanian ${ }^{1}$, Swapna Talluri ${ }^{2}$, Sanjana Mullangi ${ }^{3}$, Manidhar R. Lekkala ${ }^{4}$, Bahar Moftakhar ${ }^{4}$

1. Department of Medicine, Washington Hospital Healthcare System, Fremont, USA 2. Department of Medicine, Guthrie Robert Packer Hospital, Sayre, USA 3. Department of Medicine, Hillcrest Medical Center, Tulsa, USA 4. Department of Hematology/Oncology, James P Wilmot Cancer Institute, University of Rochester School of Medicine and Dentistry, Rochester, USA

Corresponding author: Manidhar R. Lekkala, mani.lr@outlook.com

\begin{abstract}
Bone is the most common site for distant metastases in breast cancer and can cause significant morbidity and mortality. Bone modifying agents (BMAs) that include bisphosphonates (BPAs) and denosumab help in decreasing and delaying skeletal-related events (SREs) associated with metastatic breast cancer. BPAs approved for use by the Food and Drug Administration (FDA) in bone metastases (BM) in the United States are pamidronate and zolendronic acid, while clodronate and ibandronate are licensed for use in other countries. Current American Society of Clinical Oncology (ASCO) guidelines recommend denosumab $120 \mathrm{mg}$ subcutaneously every four weeks, or zolendronic acid $4 \mathrm{mg}$ every four weeks or every 12 weeks, or intravenous pamidronate $90 \mathrm{mg}$ every four weeks. Current guidelines do not recommend one BMA over another, however, zolendronic acid and denosumab were the most commonly used BMAs in populationbased studies. Side effects of BMAs include acute phase reactions, hypocalcemia, nephrotoxicity, osteonecrosis of jaw, etc. While other side effects are common with both BPAs and denosumab, the latter has less nephrotoxic potential and is preferred for use in patients with renal failure. Current ASCO guidelines recommend continuing BMAs indefinitely, however, in clinical practice, this decision needs to be individualized, especially since there is no data on the impact of long-term use of BMAs. Further studies would need to be developed to develop an algorithm of SRE risk assessment and to determine which patients would benefit from BMAs.
\end{abstract}

Categories: Oncology

Keywords: bone modifying agents, metastatic breast cancer

\section{Introduction And Background}

Bone is the most common site for distant metastases in breast cancer [1] and can occur in up to $58 \%$ of patients with advanced disease [2]. Metastatic spread is the leading cause of breast cancer-related mortality. In this manuscript, we review the current data on the usage of bone modifying agents (BMAs) in metastatic breast cancer.

Review began 02/06/2021 Review ended 02/09/2021 Published 02/13/2021

๑) Copyright 2021

Raghu Subramanian et al. This is an open access article distributed under the terms of the Creative Commons Attribution License CC-BY 4.0., which permits unrestricted use, distribution, and reproduction in any medium, provided the original author and source are credited.

\section{Review}

\section{Mechanism of bone metastases (BM)}

BM can be osteolytic, osteoblastic, or mixed, with the majority being osteolytic in nature [3]. Osteoclast activation is the main mechanism for osteolytic metastases associated with breast cancer. When osteoclasts are activated, multiple signaling cascades are turned on that destabilize the bone matrix, leading to accelerated bone loss and creating a favorable environment for tumor growth. BMAs, such as bisphosphonates (BPAs) and receptor activator of nuclear factor kappa-B ligand (RANKL) inhibitors (e.g., denosumab), modulate osteoclastic activity to suppress these effects [4].

\section{BM in breast cancer}

BM can cause significant morbidity and mortality in patients with breast cancer and, without BMAs, 50\% of these patients can develop a skeletal-related event (SRE) [5]. SREs include hypercalcemia, spinal cord compression, pathological fracture, or the requirement of radiation or surgery for an impending fracture [5]. While women with bone-only metastatic breast cancer have a median survival of two-three years, this could be shortened to three months (hypercalcemia), four months (spinal cord compression), or 12 months (fracture), depending on the type of SRE $[1,5]$.

Current BMAs in use for metastatic breast cancer are BPAs and denosumab. BPAs delay the onset of skeletal complications by inhibiting osteoclast-mediated bone resorption [6]. They bind to the bone mineral where they are absorbed by mature osteoclasts and subsequently induce osteoclast apoptosis [7]. Denosumab is a fully human monoclonal antibody that binds and inhibits the cytokine RANKL (receptor activator of NFKB 
ligand) which blocks osteoclast maturation and survival, thus reducing bone resorption [7].

\section{BMAs used in metastatic breast cancer}

The earliest studies of BPAs use for BM involved trials of clodronate [8] and pamidronate $[9,10]$ in the early 1990s. Since then, several BPAs have been studied in various randomized controlled trials (RCTs). The BPAs pamidronate and zoledronic acid were approved for the treatment of BM by the Food and Drug Administration (FDA) in 1996 and 2002, respectively. Denosumab was approved by the FDA in 2010 [11], while clodronate and ibandronate have been licensed for use in other countries [12]. BPAs are administered either intravenously (zoledronic acid, pamidronate, ibandronate) or orally (clodronate or ibandronate). Denosumab is administered subcutaneously.

Current American Society of Clinical Oncology (ASCO) guidelines recommend BMAs for patients with breast cancer with BM who have evidence of bone destruction. The recommended agents for use are denosumab $120 \mathrm{mg}$ subcutaneously every four weeks, intravenous pamidronate $90 \mathrm{mg}$ over no less than 2 hours, or zoledronic acid $4 \mathrm{mg}$ over no less than 15 minutes given every three to four weeks or every 12 weeks [13]. The recommendation for de-escalation of the frequency of zoledronic acid to every 12 weeks was added in 2017 after three RCTs (CALBG, ZOOM, and OPTIMIZE-2) and a meta-analysis by Ibrahim et al. showed no difference in outcomes between a shorter versus longer interval between infusions [14-17]. Various RCTs have demonstrated the efficacy of BMAs in metastatic breast cancer patients and in a recent large metaanalysis, BPAs showed a reduction in SREs by $16 \%-17 \%$ and decreased median time to SREs compared to placebo. Denosumab reduced SREs by $22 \%$ when compared to BPAs. None of the agents seemed to affect survival [18].

\section{Comparison between different BMAs}

Current guidelines do not recommend one BMA over another, however, in clinical practice, some agents are favored over others. Among BPAs, zoledronic acid is preferred compared to others due to efficacy data [19]. In head to head comparisons, zoledronic acid was found to be superior to ibandronate in preventing SREs in the ZICE trial [20]. In another RCT, zoledronic acid demonstrated higher efficacy in patients with breast carcinoma than pamidronate. In a multiple-event analysis, zoledronic acid significantly lowered the mean skeletal morbidity rate, increased the median time to first SRE, and reduced the risk of developing an SRE by $30 \%$ compared to pamidronate [21]. Another advantage of zoledronic acid over pamidronate is its shorter infusion time.

Among other bisphosphonates, ibandronate has also been widely studied. It is found to be more costeffective compared to the more commonly used IV bisphosphonates, and this can be a consideration in select patients [22]. A comparison between oral and IV ibandronate showed equal efficacy [23]. Although oral treatment is more convenient, it should be used with caution given the potential for gastrointestinal (GI) side effects and lower efficacy in preventing SREs than the other IV agents [24].

When comparing BPAs to the RANKL inhibitor, denosumab, head to head trials were more favorable towards denosumab [25-27]. Stopeck et al. showed that denosumab reduced the risk of developing multiple SREs by $23 \%$ compared to zoledronic acid. Denosumab was also superior to zoledronic acid in reducing the mean skeletal morbidity rate (defined as the ratio of the number of SREs per patient divided by the patient's time at risk) [26]. Denosumab had a more favorable safety profile with less incidence of renal toxicity and acute phase reactions. Convenient subcutaneous injection and no requirement for renal monitoring with denosumab are also cited as advantages $[25,26]$. Two different meta-analyses also demonstrated denosumab to be preferable over zoledronic acid and pamidronate $[3,12]$.

In population-based studies, zoledronic acid and denosumab were the most commonly used BMAs [28]. Given the above data, some authors have questioned the guidelines not recommending one BMA over another, opining that denosumab seems to be superior to other BMAs in various aspects [29].

\section{Side effects}

Adverse events (AEs) with BMAs have been reported in trials and population studies (Table 1). These include GI-related events such as nausea and vomiting, as well as impaired renal function, acute phase reactions, electrolyte abnormalities, and osteonecrosis of jaw. Most AEs were low grade and in general, grade 3/4 AEs were rare [18]. 


\section{Cureus}

\begin{tabular}{|c|c|c|c|}
\hline \multirow{2}{*}{ Drug } & \multirow{2}{*}{ Potential Side effects } & \multicolumn{2}{|c|}{ Monitoring parameters } \\
\hline & & $\begin{array}{l}\text { Prior to initiation of } \\
\text { therapy }\end{array}$ & Prior to each dose \\
\hline $\begin{array}{l}\text { IV } \\
\text { Zoledronic } \\
\text { Acid }\end{array}$ & $\begin{array}{l}\text { Hypocalcemia Influenza like reaction Musculoskeletal } \\
\text { Pain Osteonecrosis of Jaw Atypical bone fractures } \\
\text { Ocular Side effects }\end{array}$ & $\begin{array}{l}\text { Dental Exam and } \\
\text { appropriate } \\
\text { preventative } \\
\text { dentistry }\end{array}$ & $\begin{array}{l}\text { Basic Metabolic Panel Calcium Phosphate } \\
\text { Magnesium }\end{array}$ \\
\hline $\begin{array}{l}\text { IV } \\
\text { Pamidronate }\end{array}$ & $\begin{array}{l}\text { Electrolyte abnormalities Musculoskeletal Pain Atypical } \\
\text { bone fractures Myelosuppression Osteonecrosis of Jaw }\end{array}$ & $\begin{array}{l}\text { Dental Exam and } \\
\text { appropriate } \\
\text { preventative } \\
\text { dentistry }\end{array}$ & $\begin{array}{l}\text { Basic Metabolic Panel Calcium Phosphate } \\
\text { Magnesium CBC }\end{array}$ \\
\hline $\begin{array}{l}\text { Ibandronate } \\
\text { PO }\end{array}$ & $\begin{array}{l}\text { Gl side effects Hypocalcemia Influenza like reaction } \\
\text { Musculoskeletal Pain Osteonecrosis of Jaw Atypical } \\
\text { bone fractures Ocular Side effects }\end{array}$ & $\begin{array}{l}\text { Dental Exam and } \\
\text { appropriate } \\
\text { preventative } \\
\text { dentistry }\end{array}$ & $\begin{array}{l}\text { Basic Metabolic Panel Calcium Phosphate } \\
\text { Magnesium }\end{array}$ \\
\hline $\begin{array}{l}\text { Denosumab } \\
\text { SQ }\end{array}$ & $\begin{array}{l}\text { Dermatitis, rash, eczema Hypersensitivity } \\
\text { Hypocalcemia Infections Musculoskeletal pain Atypical } \\
\text { femur fractures Osteonecrosis of Jaw }\end{array}$ & $\begin{array}{l}\text { Dental Exam and } \\
\text { appropriate } \\
\text { preventative } \\
\text { dentistry }\end{array}$ & $\begin{array}{l}\text { Basic Metabolic Panel Calcium Phosphate } \\
\text { Magnesium Signs/Symptoms of hypersensitivity } \\
\text { Signs/symptoms of dermatitis }\end{array}$ \\
\hline$=1+1+2$ & a modi & MAs $>$ & itoring parar \\
\hline
\end{tabular}

Acute-phase reactions are defined as flu-like symptoms including pyrexia, chills, flushing, bone pain, and myalgias. These events generally occur within three days of treatment and subside rapidly thereafter [26,30]. Hypocalcemia is the most common electrolyte abnormality associated with BMA therapy [30]. This has been documented to be more frequent with denosumab than zoledronic acid in comparative clinical trials $[25,26]$. While most hypocalcemia events are asymptomatic and grade 2 or less, some could be severe, and even fatal cases have been reported [30,31]. Use of calcium and vitamin D supplements has been associated with a $40 \%$ lower risk of developing hypocalcemia with denosumab and it is recommended that patients receive daily supplementation. Serum calcium and vitamin D should be checked and be within normal limits prior to the initial dose and then checked prior to each dose thereafter [32,33]. However, if hypocalcemia develops, the BMA should be stopped and the patient should be treated accordingly [30,34].

Nephrotoxicity is another well-known AE of BPAs. Both zoledronic acid and pamidronate have been associated with renal failure. There have been more reports of renal failure with zoledronic acid than pamidronate [21,34]. Although acute renal failure may be reversible, some degree of irreversible impairment may persist leading to chronic renal failure. Treatment is generally not recommended if serum creatinine is $>3.0 \mathrm{mg} / \mathrm{dl}$ or creatinine clearance is $<30 \mathrm{ml} / \mathrm{min}$ [34]. Treatment should be withheld temporarily if serum creatinine increases greater than twice the patient's baseline or greater than $0.5 \mathrm{mg} / \mathrm{dl}$ for patients with baseline $\leqslant 1.4 \mathrm{mg} / \mathrm{dl}$ and $>1 \mathrm{mg} / \mathrm{dl}$ for others [35]. Zoledronic acid can be resumed when serum creatinine returns within $10 \%$ of baseline, however, should be permanently discontinued if no improvement is seen within four-eight weeks [35]. In contrast, denosumab was reported to have less renal toxicity in comparative trials $[26,36]$. Since denosumab elimination is not reliant on renal function, it presents a therapeutic option for patients with chronic renal failure [26]. Patients with chronic renal disease are at risk of severe hypocalcemia with denosumab compared to patients with normal renal function and this would need to be monitored closely [36].

Osteonecrosis of the jaw (ONJ) is another well recognized adverse event related to both bisphosphonates and denosumab [30]. A meta-analysis of several randomized trials did not show a significant difference in the incidence of ONJ between bisphosphonates and denosumab [37]. Poor oral hygiene, dentures, dentoalveolar surgery, dental and periodontal infections, systemic factors such as smoking, diabetes, renal insufficiency, total dose, and duration of treatment have been implicated as risk factors [30,38]. It is recommended that patients undergo a dental screening exam prior to the initiation of BMAs so that any required dental procedures can be carried out in advance [34]. While on BMAs patients should maintain good oral hygiene and have dental examinations at regular intervals. It is advised to avoid dental procedures while on BMAs, however, if it is deemed necessary, then it is prudent to stop BMAs prior to the procedure and resume only after appropriate wound healing [30,34].

\section{Effect on quality of life (QoL)/pain management}

Despite the benefit of reducing and delaying the onset of SREs, none of the BMAs have shown a survival 
benefit. In patients with metastatic and incurable disease, a relevant and important aim is improved QoL, a key component of which is pain control. Weinfurt et al. showed that women with metastatic breast cancer treated with BPAs had a gradual increase in physical, functional, and emotional well-being. Subjects also reported that a previous SRE was one of the reasons for the lack of improvement in QoL [39].

Porta-Sales et al. noted that BMAs while having only a weak analgesic effect, are actually beneficial in delaying the onset of bone pain [40]. Denosumab had a significant effect on pain, and patients treated with denosumab had more pain-free periods compared to patients treated with zoledronic acid [41]. Most patients (79\%) had bone pain at the time of diagnosis of BM in a study by von Moos et al., and this was the main reason for initiating BMA [28]. Evidence suggests that early detection and treatment of BM before the onset of pain could benefit not only with pain relief but also decreased SREs [42]. BMAs' analgesic effects however are modest and per ASCO guidelines, should not be used as the primary means of pain control.

\section{When to start therapy/duration}

The incidence of SREs is highest during the first year after the diagnosis of metastatic breast cancer. A Danish-based population study estimated that the cumulative incidence of SREs in patients with metastatic breast cancer was $38.5 \%$ at one year and $51.7 \%$ at five years [43]. Appropriately, in recent population-based studies, most patients with BMs were treated with BMAs early in their treatment course [28].

Current ASCO guidelines recommend continuing treatment with BMAs indefinitely [44] unless toxicity occurs or patient preference changes. If a breakthrough SRE occurs while on treatment with a BMA, it is generally advised to continue BMAs as they can lengthen the time to subsequent SREs [21,45]. There are no current guidelines for changing from one BMA to another. The patient could either continue with the same BMA or consider a switch. Denosumab could be a reasonable alternative if the patient was on an IV bisphosphonate earlier since it has been proven to be successful in patients previously treated with IV BPAs [25].

In clinical practice, the decision to treat indefinitely would need to be individualized. Several studies of realworld data show that patients experienced fewer SREs than patients enrolled on RCTs. This is thought to be due to a large proportion of patients who have both visceral and BM, and unlike in the bone-only disease population, as seen in these studies, the former have shorter survival time and thus less opportunity for SREs or fractures in their lifespan $[5,46]$. Gainford et al. also observed that patients in clinical practice do not undergo serial imaging if they are asymptomatic in contrast to patients in RCTs who undergo X-rays at regular intervals [5]. This was also elucidated in other population studies where around $30 \%$ of patients developed clinically significant SREs, while in RCTs, these numbers were in the 40s and 50s (treatment and placebo arms respectively) $[1,47]$. Further studies will be necessary to develop an algorithm of SRE risk assessment and to determine which patients would benefit from BMAs.

Age $>60$ years, previous SRE, a predominance of osteolytic lesions, and brief pain inventory score $>3$ units are some of the parameters identified in previous studies that have demonstrated an increased risk of SRE [48]. Machine learning models to predict SREs are also being studied, and if developed, could be used in metastatic breast cancer cases [49].

\section{Future studies/trials}

The ongoing trials, REDUSE and REaCT-BTA, are studying different dosing intervals of denosumab (four weeks vs twelve weeks) and results are currently being awaited [13]. There are no RCTs to determine the duration of treatment for BMAs. There is a need for these RCTs as well as a need for more data on the impact of long-term use of BMAs.

\section{Conclusions}

In conclusion, BMAs play an important role in the treatment of breast cancer patients with BM. Based on RCT results and real-world data, zoledronic acid and denosumab are favored compared to other BMAs. While denosumab has shown slight superiority in RCTs in reducing the rate of SREs and delaying the onset of SREs, zoledronic is also safe, efficacious, and importantly, more cost-effective than the former. Denosumab is preferred in patients with chronic renal failure or those who are also receiving nephrotoxic chemotherapeutic agents. As described above, more research is needed to identify patients who would benefit from BMAs, to determine the optimal duration of treatment, and to elucidate long-term effects.

\section{Additional Information \\ Disclosures}

Conflicts of interest: In compliance with the ICMJE uniform disclosure form, all authors declare the following: Payment/services info: All authors have declared that no financial support was received from any organization for the submitted work. Financial relationships: All authors have declared that they have no financial relationships at present or within the previous three years with any organizations that might have an interest in the submitted work. Other relationships: All authors have declared that there are no 


\section{References}

1. Coleman RE, Rubens RD: The clinical course of bone metastases from breast cancer . Br J Cancer. 1987, 55:61-6. 10.1038/bjc.1987.13

2. Body JJ, Quinn G, Talbot S, Booth E, Demonty G, Taylor A, Amelio J: Systematic review and meta-analysis on the proportion of patients with breast cancer who develop bone metastases. Crit Rev Oncol Hematol. 2017, 115:67-80. 10.1016/j.critrevonc.2017.04.008

3. Tesfamariam Y, Jakob T, Wöckel A, et al.: Adjuvant bisphosphonates or RANK-ligand inhibitors for patients with breast cancer and bone metastases: a systematic review and network meta-analysis. Crit Rev Oncol Hematol. 2019, 137:1-8. 10.1016/j.critrevonc.2019.02.004

4. Heeke A, Nunes MR, Lynce F: Bone-modifying agents in early-stage and advanced breast cancer . Curr Breast Cancer Rep. 2018, 10:241-50. 10.1007/s12609-018-0295-6

5. Gainford MC, Dranitsaris G, Ooi W, Vanhuyse M, Clemons M: Comparing the results of bisphosphonate use in clinical trials with actual practice: a case of apples and oranges?. Curr Oncol. 2006, 13:187-90.

6. Luo Q, Men P, Liu Z, Zhai S, Jiang M: Is de-escalated bisphosphonates therapy a suitable alternative to standard dosing in malignant tumor patients with bone metastases: a systematic review and meta-analysis. Front Oncol. 2019, 9:774. 10.3389/fonc.2019.00774

7. Hanley DA, Adachi JD, Bell A, Brown V: Denosumab: mechanism of action and clinical outcomes . Int J Clin Pract. 2012, 66:1139-46. 10.1111/ijcp.12022

8. Paterson AHG, Powles TJ, Kanis JA, McCloskey E, Hanson J, Ashley S: Double-blind controlled trial of oral clodronate in patients with bone metastases from breast cancer. J Clin Oncol. 1993, 11:59-65. 10.1200/JCO.1993.11.1.59

9. Conte PF, Latreille J, Mauriac L, et al.: Delay in progression of bone metastases in breast cancer patients treated with intravenous pamidronate: Results from a multinational randomized controlled trial. J Clin Oncol. 1996, 14:2552-9. 10.1200/JCO.1996.14.9.2552

10. Van Holten-Verzantvoort ATM, Kroon HM, Bijvoet OLM, et al.: Palliative pamidronate treatment in patients with bone metastases from breast cancer. J Clin Oncol. 1993, 11:491-8. 10.1200/JCO.1993.11.3.491

11. Food and Drug Administration: Xgeva (denosumab). (2020). Accessed: November 23, 2020: https://www.accessdata.fda.gov/drugsatfda_docs/label/2013/125320s094lbl.pdf.

12. Wang Z, Qiao D, Lu Y, Curtis D, Wen X, Yao Y, Zhao H: Systematic literature review and network metaanalysis comparing bone-targeted agents for the prevention of skeletal-related events in cancer patients with bone metastasis. Oncologist. 2015, 20:440-9. 10.1634/theoncologist.2014-0328

13. Van Poznak C, Somerfield MR, Barlow WE, et al.: Role of bone-modifying agents in metastatic breast cancer: an American Society of Clinical oncology-cancer Care Ontario focused guideline update. J Clin Oncol. 2017, 35:3978-86. 10.1200/JCO.2017.75.4614

14. Amadori D, Aglietta M, Alessi B, et al.: Efficacy and safety of 12-weekly versus 4-weekly zoledronic acid for prolonged treatment of patients with bone metastases from breast cancer (ZOOM): a phase 3, open-label, randomised, non-inferiority trial. Lancet Oncol. 2013, 14:663-70. 10.1016/S1470-2045(13)70174-8

15. Himelstein AL, Foster JC, Khatcheressian JL, et al.: Effect of longer-interval vs standard dosing of zoledronic acid on skeletal events in patients with bone metastases: a randomized clinical trial. JAMA. 2017, 317:48-58. 10.1001/jama.2016.19425

16. Hortobagyi GN, Van Poznak C, Harker WG, et al.: Continued treatment effect of zoledronic acid dosing every 12 vs 4 weeks in women with breast cancer metastatic to bone: the OPTIMIZE-2 randomized clinical trial. JAMA Oncol. 2017, 3:906-12. 10.1001/jamaoncol.2016.6316

17. Ibrahim MFK, Mazzarello S, Shorr R, et al.: Should de-escalation of bone-targeting agents be standard of care for patients with bone metastases from breast cancer? A systematic review and meta-analysis. Ann Oncol. 2015, 26:2205-13. 10.1093/annonc/mdv284

18. O'Carrigan B, Wong MHF, Willson ML, Stockler MR, Pavlakis N, Goodwin A: Bisphosphonates and other bone agents for breast cancer. Cochrane Database Syst Rev. 2017, CD003474. 10.1002/14651858.CD003474.pub4

19. Palmieri C, Fullarton JR, Brown J: Comparative efficacy of bisphosphonates in metastatic breast and prostate cancer and multiple myeloma: a mixed-treatment meta-analysis. Clin Cancer Res. 2013, 19:686372. 10.1158/1078-0432.CCR-13-2275

20. Barrett-Lee P, Casbard A, Abraham J, et al.: Oral ibandronic acid versus intravenous zoledronic acid in treatment of bone metastases from breast cancer: a randomised, open label, non-inferiority phase 3 trial. Lancet Oncol. 2014, 15:114-22. 10.1016/S1470-2045(13)70539-4

21. Rosen LS, Gordon D, Kaminski M, et al.: Long-term efficacy and safety of zoledronic acid compared with pamidronate disodium in the treatment of skeletal complications in patients with advanced multiple myeloma or breast carcinoma. Cancer. 2003, 98:1735-44. 10.1002/cncr.11701

22. De Cock E, Hutton J, Canney P, Body JJ, Barrett-Lee P, Neary MP, Lewis G: Cost-effectiveness of oral ibandronate compared with intravenous (i.v.) zoledronic acid or i.v. generic pamidronate in breast cancer patients with metastatic bone disease undergoing i.v. chemotherapy. Support Care Cancer. 2005, 13:975-86. 10.1007/s00520-005-0828-1

23. Mystakidou K, Stathopoulou E, Parpa E, Kouloulias V, Kouskouni E, Vlahos L: Oral versus intravenous ibandronic acid: a comparison of treatment options for metastatic bone disease. J Cancer Res Clin Oncol. 2008, 134:1303-10. 10.1007/s00432-008-0419-x

24. von Au A, Milloth E, Diel I, et al.: Intravenous pamidronate versus oral and intravenous clodronate in bone metastatic breast cancer: a randomized, open-label, non-inferiority phase III trial. Onco Targets Ther. 2016, 9:4173-80. 10.2147/OTT.S103130

25. Fizazi K, Lipton A, Mariette X, et al.: Randomized phase II trial of denosumab in patients with bone metastases from prostate cancer, breast cancer, or other neoplasms after intravenous bisphosphonates. J Clin Oncol. 2009, 27:1564-71. 10.1200/JCO.2008.19.2146 
26. Stopeck AT, Lipton A, Body JJ, et al.: Denosumab compared with zoledronic acid for the treatment of bone metastases in patients with advanced breast cancer: a randomized, double-blind study. J Clin Oncol. 2010, 28:5132-9. 10.1200/JCO.2010.29.7101

27. Lipton A, Steger GG, Figueroa J, et al.: Extended efficacy and safety of denosumab in breast cancer patients with bone metastases not receiving prior bisphosphonate therapy. Clin Cancer Res. 2008, 14:6690-6. 10.1158/1078-0432.CCR-07-5234

28. von Moos R, Body JJ, Rider A, et al.: Bone-targeted agent treatment patterns and the impact of bone metastases on patients with advanced breast cancer in real-world practice in six European countries. J Bone Oncol. 2018, 11:1-9. 10.1016/j.jbo.2017.11.004

29. Gralow JR, Lipton A, Stopeck AT: How do we define efficacy in comparing osteoclast-targeted agents in metastatic breast cancer?. J Clin Oncol. 2011, 29:3591-2. 10.1200/JCO.2011.36.5759

30. Domschke C, Schuetz F: Side effects of bone-targeted therapies in advanced breast cancer . Breast Care. 2014, 9:332-6. 10.1159/000368844

31. Body JJ, Bone HG, de Boer RH, et al.: Hypocalcaemia in patients with metastatic bone disease treated with denosumab. Eur J Cancer. 2015, 51:1812-21. 10.1016/j.ejca.2015.05.016

32. Pittman K, Antill YC, Goldrick A, Goh J, de Boer RH: Denosumab: prevention and management of hypocalcemia, osteonecrosis of the jaw and atypical fractures. Asia Pac J Clin Oncol. 2017, 13:266-76. 10.1111/ajco.12517

33. FDA: highlights of prescribing information . (2010). Accessed: December 10, 2020: https://www.accessdata.fda.gov/drugsatfda_docs/label/2013/125320s094lbl.pdf.

34. Tanvetyanon T, Stiff PJ: Management of the adverse effects associated with intravenous bisphosphonates . Ann Oncol. 2006, 17:897-907. 10.1093/annonc/mdj105

35. Berenson JR: Recommendations for zoledronic acid treatment of patients with bone metastases . Oncologist. 2005, 10:62. 10.1634/theoncologist.10-1-52

36. Prommer E: Palliative oncology: denosumab. Am J Hosp Palliat Med. 2015, 32:568-72.

10.1177/1049909114539035

37. Wang X, Yang KH, Wanyan P, Tian JH: Comparison of the efficacy and safety of denosumab versus bisphosphonates in breast cancer and bone metastases treatment: a meta-analysis of randomized controlled trials. Oncol Lett. 2014, 7:1997-2002. 10.3892/ol.2014.1982

38. Campisi G, Fedele S, Fusco V, Pizzo G, Di Fede O, Bedogni A: Epidemiology, clinical manifestations, risk reduction and treatment strategies of jaw osteonecrosis in cancer patients exposed to antiresorptive agents. Futur Oncol. 2014, 10:257-75. 10.2217/fon.13.211

39. Weinfurt KP, Castel LD, Li Y, Timbie JW, Glendenning GA, Schulman KA: Health-related quality of life among patients with breast cancer receiving zoledronic acid or pamidronate disodium for metastatic bone lesions. Med Care. 2004, 42:164-75. 10.1097/01.mlr.0000108746.69256.45

40. Porta-Sales J, Garzón-Rodríguez C, Llorens-Torromé S, Brunelli C, Pigni A, Caraceni A: Evidence on the analgesic role of bisphosphonates and denosumab in the treatment of pain due to bone metastases: a systematic review within the European Association for Palliative Care guidelines project. Palliat Med. 2017, 31:5-25. 10.1177/0269216316639793

41. Stopeck A, Fallowfield L, Patrick D, et al.: Effects of denosumab versus zoledronic acid (ZA) on pain in patients (pts) with metastatic breast cancer: Results from a phase III clinical trial. J Clin Oncol. 2010, 28:1024-1024. 10.1200/jco.2010.28.15_suppl.1024

42. Costa L, Lipton A, Hadji P, Chen YM, Kosmidis P: Treatment of bone metastases before the onset of pain . Int J Clin Oncol. 2013, 18:531-8. 10.1007/s10147-012-0414-8

43. Jensen AT, Jacobsen JB, Nørgaard M, Yong M, Fryzek JP, Sørensen HT: Incidence of bone metastases and skeletal-related events in breast cancer patients: a population-based cohort study in Denmark. BMC Cancer. 2011, 11:29. 10.1186/1471-2407-11-29

44. Van Poznak CH, Temin S, Yee GC: American Society of Clinical Oncology Clinical Practice Guideline update on the role of bone-modifying agents in metastatic breast cancer. J Oncol Pract. 2011, 7:117-21. 10.1200/jop.2011.000212

45. Plunkett TA, Smith P, Rubens RD: Risk of complications from bone metastases in breast cancer: implications for management. Eur J Cancer. 2000, 36:476-82. 10.1016/S0959-8049(99)00331-7

46. Liauw W, Segelov E, Lih A, Dunleavy R, Links M, Ward R: Off-trial evaluation of bisphosphonates in patients with metastatic breast cancer. BMC Cancer. 2005, 5:89. 10.1186/1471-2407-5-89

47. Van Poznak C: Predicting skeletal complications in metastatic breast cancer raises challenges . Breast Cancer Res Treat. 2010, 123:781-3. 10.1007/s10549-010-1104-8

48. Wang Z, Wen X, Lu Y, Yao Y, Zhao H: Exploiting machine learning for predicting skeletal-related events in cancer patients with bone metastases. Oncotarget. 2016, 7:12612-22. 10.18632/oncotarget.7278

49. Shapiro CL, Moriarty JP, Dusetzina S, et al.: Cost-effectiveness analysis of monthly zoledronic acid, zoledronic acid every 3 months, and monthly denosumab in women with breast cancer and skeletal metastases: CALGB 70604 (Alliance). J Clin Oncol. 2017, 35:3949-55. 10.1200/JCO.2017.73.7437 